\title{
The Relationship between Active Magnetic Bearing System's Stiffness and Bias Current
}

\author{
Jiong Wang ${ }^{1, a^{*}}$, Hu Cheng ${ }^{1, b}$, Hourao Luo ${ }^{1, c}$ \\ ${ }^{1}$ College of Mechatronic Engineering and Automation,National University of Defense \\ Technology, Changsha, Hunan, China \\ awangjiong339@126.com, b13875851753@163.com, c407639648@qq.com,
}

Keywords: Active magnetic bearing, PD control, bearing stiffness, bias current.

Abstract. The paper established a single-degree-of-freedom model for Active magnetic bearing (AMB) system, on the purpose to analyze the stability range of parameters for AMB close-loop system under the control of PD. Furthermore, it focused on the study of relationship between the AMB system's stiffness and the bias current under stable system. Finally, it presented analysis of a simulation example,to compare and verify the theoretical deduction. The results showed that, if other parameters remained unchanged, AMB close-loop system`s stiffness was a quadratic function which had bias current as an independent variable. However, the mere increment of bias current did not increase the stiffness of AMB system without boundary, but there existed a maximum stiffness for a certain bias current. In conclusion, the results of this paper provided an appropriate bias current for AMB system, which assisted AMB system to obtain better dynamic performance.

\section{Introduction}

Active magnetic bearing(AMB) is a newly developed non-contact and high-performance bearing which is based on the use of electromagnetic force to keep rotor suspending. Compared to the traditional bearing, AMB has several benefits such as less degree of mechanical wear, longer service life, no use for lubrication, etc. Regular magnetic bearing is adopting bias current control method, which is, with superposition(addition or subtraction) of the bias current and the control current, creating a proper difference of electromagnetic force between them, to maintain equilibrium position of rotor. This approach allows bearing system with a high stiffness and stability. But the bias current also is the reason to cause higher magnetic field strength and stronger copper and iron damage. Therefore, developing the control method of variable bias or zero bias current, is an important way to reduce the loss of the active magnetic bearing system.

There are massive foreign research concerning variable bias current control strategy. N. Motee proposed a nonlinear control strategy which reduced the amount of bias current by index approach, and provided a faster speed to stabilize the closed loop system [1]. Shailesh Pradhananga studied the variable bias current control for magnetic suspending flywheel, then revealed that the bias current changed with time. The experimental results also showed that such method reduced system loss while without affecting stability [2]. Nobuyuki Kobayashi used the bias current to adjust the the additional stiffness of AMB system, in order to decrease system loss [3].Dexter Johnson came to the idea of adopting an adaptive PD control method to realize variable bias current control based on AMB system [4]. Besides, several domestic scholars also made important contributions to the study. Kaifeng $\mathrm{Wu}$ designed and created zero-bias axial controller, five degree of freedom simulating controller with zero bias, and digital variable bias controller, and carried experiment which showed that the system was able to reduce the total current consumption and at the same time not to deteriorate the dynamic performance [5]. Another paper [6] discussed when introducing session of measuring speed with the F28335DSP controller, then choosing varying bias current according to different speed, while through $H_{\infty}$ and PID control strategy, how could the zero-bias and variable bias current method affect the static and dynamic performance of the AMB system. It concluded that the size of the bias current had an influence on its dynamic performance. However, the research above lacked specific analysis of the relationship between the stiffness of AMB system and bias current. Therefore, in order to fill the scientific and technological gap, this paper, on the basis of the study 
above, established a mathematical model of single-degree-of-freedom magnetic bearing system for AMB system. As followed, it provided a calculation and analysis of the relationship between the stiffness of closed-loop AMB system and the bias current which was based on PD control, and presented a way to select a suitable bias current not only to reduce power consumption but also to assist AMB system to obtain better dynamic performance.

\section{The mathematical model of single-degree-of-freedom AMB system}

Fig. 1 is the schematic diagram of a single-degree-of-freedom AMB system. As showed in Fig. 1, we define $i_{0}$ as the bias current, $i_{c}$ as the control current, $x_{r}$ as the reference position, and $x_{s}$ as the rotor position detected by the sensors. Generally speaking, AMB is a differential structure on the single-degree-of-freedom level . i.e. The bias magnetic field generated by the bias current $i_{0}$ and the control magnetic field generated by the control current $i_{c}$ are superimposed on the magnetic field from both sides which is generated by the electromagnet to achieve a magnetic field difference.

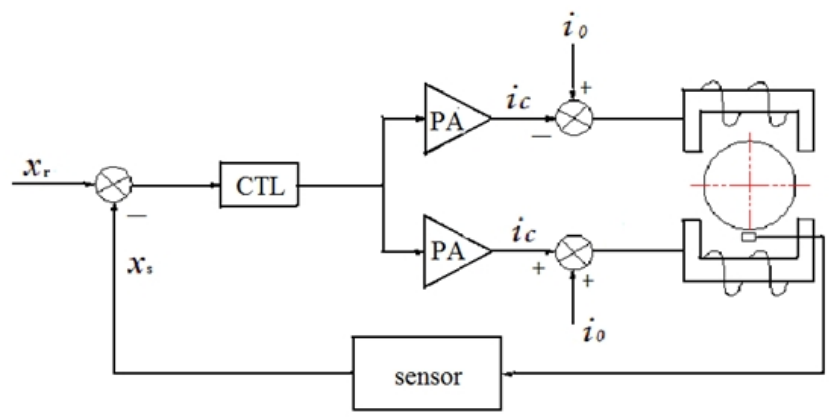

Fig.1 Schematic diagram of single-degree-of-freedom AMB system

When the bias current is not zero, the magnetic force can be expressed as below[7].

$$
F=\frac{\mu_{0} A_{0} N^{2}}{4}\left[\frac{\left(i_{0}+i_{c}\right)^{2}}{\left(x_{0}+x\right)^{2}}-\frac{\left(i_{0}-i_{c}\right)^{2}}{\left(x_{0}-x\right)^{2}}\right]
$$

Where $\mathrm{x}$ is the displacement of the rotor relative to the equilibrium position, $\mathrm{F}$ is magnetic force, which is in an opposite direction compared to $\mathrm{x}, x_{0}$ is the nominal clearance, $\mathrm{m}$ is mass of rotor, $\mu_{0}$ is the vacuum magnetic permeability, $A_{0}$ is the pole area of the electromagnet, $\mathrm{N}$ is the number of turns.

Make a Taylor expansion to (1) in the vicinity of the equilibrium point where $x=0$ and $i_{c}=0$, and omit the higher order infinitesimal, then we have

$$
F=k_{i} i_{c}-k_{x} x
$$

Equation (2) is the linearized magnetic force expression in a small deviation range. where $k_{x}$ is the displacement stiffness and $k_{x}=\frac{\mu_{0} A_{0} N^{2} i_{0}^{2}}{x_{0}^{3}}, k_{i}$ is the current stiffness and $k_{i}=\frac{\mu_{0} A_{0} N^{2} i_{0}}{x_{0}^{2}}$.

Assume the disturbing force is zero, and ignore the influence of the gravity of the rotor, we can obtain the equation below by Newton's law

$m \ddot{x}=-F$

Combine (2) and (3), the equation (3) turns to

$$
m \ddot{x}=k_{x} x-k_{i} i_{c}
$$


Set the current $I$ as the input and the rotor displacement $X$ as the output. Make a Laplace transform to (4), we obtain the transfer function of the single-degree-of-freedom AMB system

$$
G(s)=\frac{X(s)}{I(s)}=\frac{k_{i}}{m s^{2}-k_{x}}
$$

As can be seen from (5), the transfer function is a unstable second-order model. In order to stabilize the AMB system, it is required to adopt the closed-loop control.

\section{PD closed-loop control system}

According to the analysis above, we know AMB is an inherently unstable object which requires a close-loop control to maintain steady. So we introduce PD controller which is simple and popular. The PD controller takes the form of :

$$
G_{c}(s)=K_{p}+K_{d} s
$$

Where $K_{p}$ is the proportionality parameter and $K_{d}$ is the differential parameter.

Fig. 2 is the block diagram of the PD closed-loop control system. where $X_{\text {ref }}(s)$ is the reference signal of the position, $E(s)$ is the position deviation, $k_{1}$ is the gain of the power amplifier, $k_{2}$ is the gain of the position sensor, $P(s)$ is the disturbance force. According to Fig.2, we can obtain the transfer function of the closed-loop system :

$$
\phi(s)=\frac{k_{1} G_{c}(s) G(s)}{1+k_{1} k_{2} G_{c}(s) G(s)}
$$



Fig.2 Block diagram of the PD closed-loop control system

Substitute for (7) from (5) and (6), we have

$$
\phi(s)=\frac{k_{1} k_{i} K_{p}\left(1+\tau_{d} s\right)}{m s^{2}+k_{1} k_{2} k_{i} K_{d} s+k_{1} k_{2} k_{i} K_{p}-k_{x}}
$$

From function (8), we know the PD closed-loop control system of AMB is as well a second-order system. By the Rolls criterion, the necessary and sufficient conditions for the system to keep stable is that the closed-loop characteristic polynomial coefficients must be greater than zero, that is

$$
\left\{\begin{array}{l}
k_{1} k_{2} k_{i} K_{d}>0 \\
k_{1} k_{2} k_{i} K_{p}-k_{x}>0
\end{array}\right.
$$

Simplify the set of inequalities (9) and we can obtain the necessary and sufficient conditions 


$$
\left\{\begin{array}{l}
K_{d}>0 \\
K_{p}>\frac{i_{0}}{k_{1} k_{2} x_{0}}
\end{array}\right.
$$
is.

It shows that, the larger the bias current is, the smaller the stability range of the control parameters

The relationship between the bias current and the bearing stiffness

Calculation of the bearing stiffness. As mentioned before, The AMB system adopts the PD control strategy, and the transfer function of PD controller is $G_{c}(s)$. As the block diagram shown in Fig.2, we set $X_{\text {ref }}(s)=0$, then we have

$$
I_{c}(s)=k_{1} k_{2} G_{c}(s) X(s)
$$

Substitute for (11) from (6), and then make a inverse Laplace transform. We can obtain the equation below when the initial conditions is zero.

$$
i_{c}(t)=k_{1} k_{2} K_{p} x(t)+k_{1} k_{2} K_{d} \dot{x}(t)
$$

Combine (12) and (2), then we have

$$
F\left(i_{c}, x\right)=\left(k_{1} k_{2} k_{i} K_{p}-k_{x}\right) x(t)+k_{1} k_{2} k_{i} K_{d} \dot{x}(t)
$$

And we can obtain the magnetic bearing stiffness

$$
K=\frac{\partial F}{\partial x}=k_{1} k_{2} k_{i} K_{p}-k_{x}
$$

As can be known from (14), when the $k_{1}$ and $k_{2}$ are constant, the bearing stiffness is related to $k_{i}, k_{x}$ and $K_{p}$. The bigger the $K_{p}$ or $k_{i}$ is, the higher the bearing stiffness is. But on the contrary, the bigger the $k_{x}$ is, the lower the bearing stiffness is.

Relationship between bearing stiffness and the bias current. Substitute for (14) from $k_{x}=\frac{\mu_{0} A N^{2} i_{0}^{2}}{x_{0}^{3}}$ and $k_{i}=\frac{\mu_{0} A N^{2} i_{0}}{x_{0}^{2}}$, we have

$$
K=k_{1} k_{2} K_{p} \frac{\mu_{0} A N^{2}}{x_{0}^{2}} i_{0}-\frac{\mu_{0} A N^{2}}{x_{0}^{3}} i_{0}^{2}
$$

It is obvious to notice that from equation (15), when the other parameters are constant, the bearing stiffness $\mathrm{K}$ is a quadratic function of the bias current $i_{0}$. And when $i_{0}<\frac{k_{1} k_{2} K_{p} x_{0}}{2}, \mathrm{~K}$ increases with the increase of $i_{0}$. When $i_{0}>\frac{k_{1} k_{2} K_{p} x_{0}}{2}, \mathrm{~K}$ decreases with the increase of $i_{0}$. When $i_{0}=\frac{k_{1} k_{2} K_{p} x_{0}}{2}, \mathrm{~K}$ achieves the maximum value.

The reason that there exists such a relationship between $\mathrm{K}$ and $i_{0}$ is because the current stiffness $k_{i}$ and the displacement stiffness $k_{x}$ are both related to the bias current $i_{0}$. And both $k_{i}$ and $k_{x}$ are increasing as $i_{0}$ raises. But $k_{x}$ is actually a negative stiffness. The increment of $k_{x}$ will reduce the 
stiffness of the system and the system tends to be unstable. However, rather than increasing the stiffness of AMB system without boundary by raising $i_{0}$, there will be a maximum point.

\section{Simulation and calculation of the example}

Calculation of the example. We have theoretically analyzed the relationship between the magnetic bearing stiffness and the bias current above. In order to verify the assumption, we make a calculation of a specific example. The parameters of the magnetic bearing are shown in the table 1.

Table 1 Magnetic bearing system parameters

\begin{tabular}{cc}
\hline Parameters & Value \\
\hline Turns & 1500 \\
Amplifier gain & 0.5 \\
Displacement sensor gain & 1500 \\
Rotor mass $(\mathrm{kg})$ & 2.69 \\
Nominal clearance $(\mathrm{mm})$ & 0.5 \\
Pole area $\left(\mathrm{mm}^{2}\right)$ & 100 \\
\hline
\end{tabular}

Set the bias current as $i_{0}$ and substitute the parameters above for $k_{i}$ and $k_{x}$, then we can express $k_{i}$ and $k_{x}$ by $i_{0}$ :

$$
\left\{\begin{array}{l}
k_{i}=1130.4 i_{0} \\
k_{x}=2260800 i_{0}^{2}
\end{array}\right.
$$

According to (10), the stability conditions for the system can be express as :

$$
\left\{\begin{array}{l}
K_{d}>0 \\
K_{p}>2.67 i_{0}
\end{array}\right.
$$

Set $0<i_{0}<3 A, K_{p}=12$ and $K_{d}=0.01$, which match the stability conditions. Substitute the value of $K_{p}$ and $K_{d}$ to equation (15), and we have

$$
K=10209600 i_{0}-2260800 i_{0}^{2}
$$

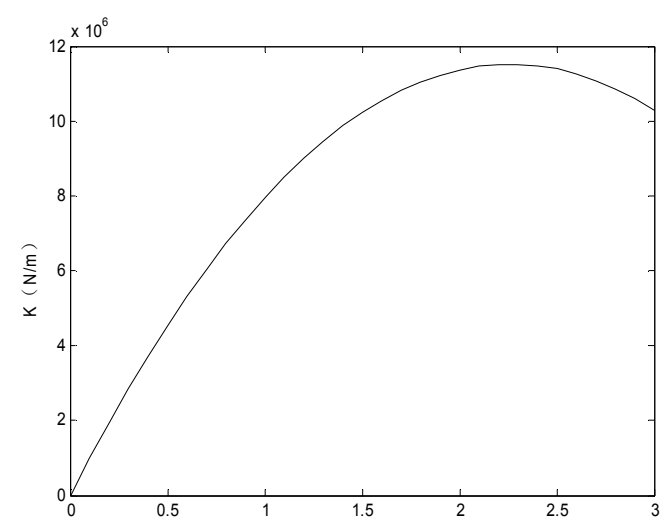

Fig. 3 The magnetic bearing stiffness curve

Fig. 3 shows the magnetic bearing stiffness curve according to (18). We can see that from the curve, the bearing stiffness increases at the beginning and then decreases. When $i_{0}=2.26 \mathrm{~A}$, the stiffness achieves the maximum value $K_{\max }=1.15 \times 10^{7} \mathrm{~N} / \mathrm{m}$. 
Simulation analysis. As showed in Fig. 4 , we build a simulation block diagram of the PD closed-loop control system by Simulink.

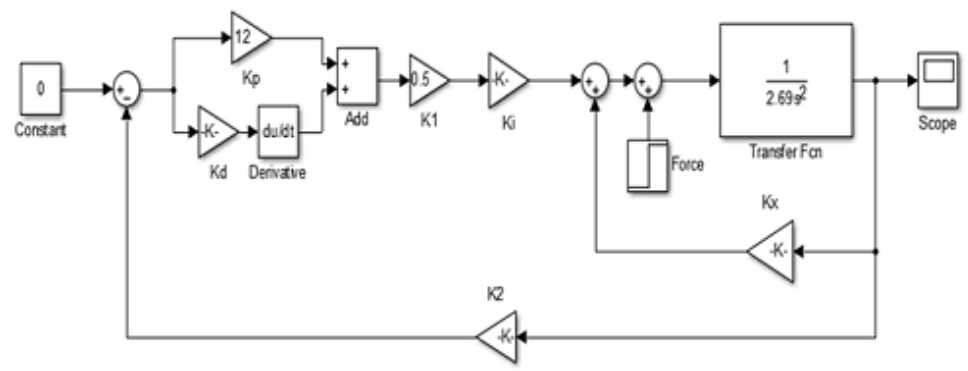

Fig.4 Simulation block diagram of the PD closed-loop control system

Set the bias current $i_{0}=1 A$, disturbance force $P(x)=1 N$. The system response curves under different $K_{p}$ are showed in Fig.5.

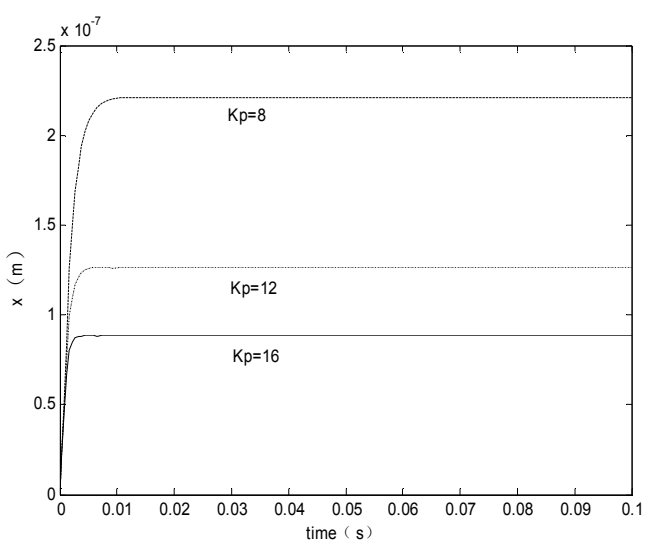

Fig.5 The system response curves under different $\mathrm{Kp}$

The result shows that, when the bias current $i_{0}$ are constant, the bigger the $K_{P}$ is, the smaller the steady-state offset under the certain disturbance force is, which means the bigger the $K_{P}$ is, the higher the bearing stiffness is.

Set the control parameters $K_{p}=12$ and $K_{d}=0.01$, disturbance force $P(x)=1 N$. The system response curves under different bias currents are showed in Fig.6.

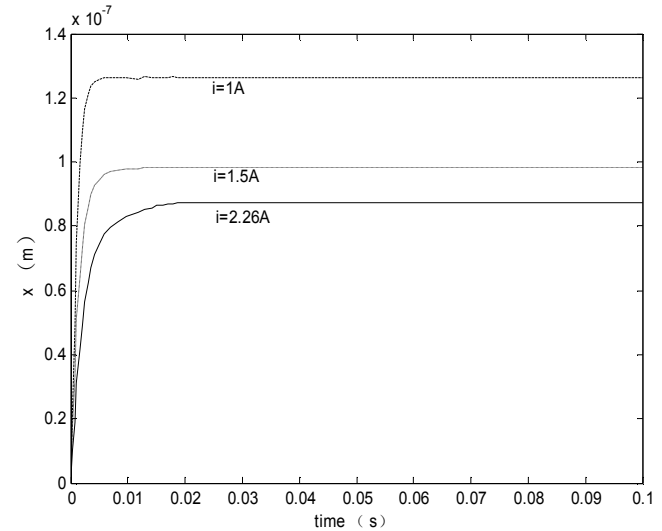

(a)

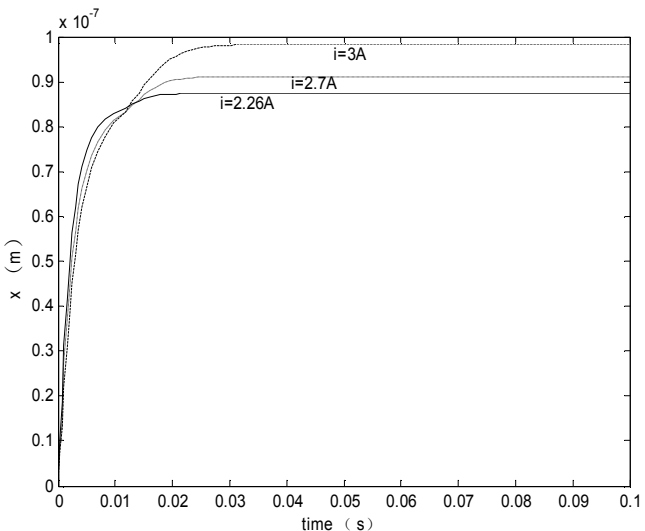

(b)

Fig.6 The system response under different bias current

The results show that, when the $K_{p}$ is constant, the steady-state offset decreases with the increase of the bias current while $i_{0}<2.26 \mathrm{~A}$, and the steady-state offset increases with the increase of the bias current while $i_{0}>2.26 \mathrm{~A}$. The steady-state offset achieves the minimum value when $i_{0}=2.26 \mathrm{~A}$. That means as the current increases, the bearing stiffness at first increases, then decreases, and achieves the maximum value when $i_{0}=2.26 \mathrm{~A}$. 


\section{Conclusions}

The paper derived the relationship between the magnetic bearing stiffness and the bias current based on PD control closed-loop system. The result showed that, the stiffness of AMB system did not increase without boundary as bias current raised, and there was a maximum value.

After simulating and analyzing how did system response under certain control parameters but different bias currents, we concluded that the simulation results were consistent with theoretical analysis above, what is more, it assisted to provide an theoretical basis on selecting the appropriate bias current for AMB system.

\section{References}

[1] N. Motee, M.S. de Queirozt. Control of Active Magnetic Bearings with a Smart Bias[C]. Proceeding of the 41st IEEE conference on decision and control,2002,1(1): 860 865.

[2] Shailesh Pradhananga. Experiment validation of a smart bias active magnetic bearing controller [D]. India:B.S.,Regional Institute of Technology,1996.

[3] Yajun Zhang, Nobuyuki Kobayashi. Aseismic control to pass through critical speed of energy storage flywheel by sliding mode control[C]. Proceedings of the 25th IASTED international conference on Modeling, identification, and control.2006,594(3)143 148.

[4] Dexter Johnson,G. V. Brown. Adaptive variable bias magnetic bearing control[C]. American control conference, 1998.

[5] Kaifeng Wu. Digital control of the adjustable bias current magnetic levitation system[D]. Nanjing: Nanjing University of Aeronautics and Astronautics, 2011.(in Chinese)

[6] Yawen Long .Control strategy of active magnetic bearing system based on variable bias current control[D]. Nanjing: Nanjing University of Aeronautics and Astronautics, 2012.(in Chinese)

[7] Yefa Hu, Zude Zhou, Zhengfeng Jiang. Basic theory and application of magnetic bearings[M]. Beijing: Mechanical Industry Press , 2006.(in Chinese) 